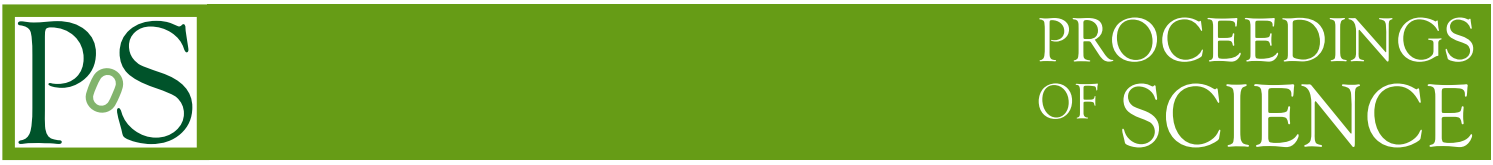

\title{
NA61/SHINE ion program
}

\author{
P. Staszel ${ }^{* \dagger}$ \\ Smoluchowski Inst. of Physics, Jagiellonian University, ul. Reymonta 4, 30-059 Kraków, Poland \\ E-mail: ufstasze@if.uj.edu.pl
}

\begin{abstract}
NA61/SHINE at the SPS facility is the successor of the former NA49 experiment. The aim of the new project is to explore the phase diagram of strongly interacting matter within the range of thermodynamical variables (like e.g. temperature and baryon chemical potential) where QCD predicts the existence of a 1-st order phase boundary between hadronic and partonic phases and the critical end point.

The detector and data acquisition system upgrades have resulted in an increase of the data rate by factor about 10 as compared to the standard NA49 data rate. These new conditions allows to perform a $2 \mathrm{D}$ scan of the phase diagram by varying energy $(10 \mathrm{~A}-158 \mathrm{~A} \mathrm{GeV})$ and size of the colliding nuclear systems $(\mathrm{p}+\mathrm{p}, \mathrm{p}+\mathrm{Pb}, \mathrm{B}+\mathrm{C}, \mathrm{Ar}+\mathrm{Ca}, \mathrm{Xe}+\mathrm{La})$. The main goal of the scan is a search for the critical point and a detailed study of the onset of QGP formation by measuring the dynamical event-by-event fluctuations, the azimuthal anisotropy as well as the inclusive pion and strange hadron production. The increase in the data rate will also give a unique possibility to measure the inclusive and correlated yields of high $p_{T}$ hadrons.
\end{abstract}

35-th International Conference on High Energy Physics,

July 22 - 282010

Paris, France

*Speaker.

${ }^{\dagger}$ for the NA61/SHINE collaboration. 
In the last decades, collisions of heavy ions in the laboratory became an important tool for studying the phase diagram of strongly interacting matter. Depending on the incident energy a highly compressed baryon dominated system or an extremely hot mesonic phase is created in these collisions. The statistical model analysis of particle yields measured in heavy-ion collisions at SIS, AGS, SPS and RHIC have been successfully used to describe the chemical freeze-out in nucleus-nucleus interactions. Some of these results show a remarkable picture at low baryochemical potential where the curves of temperature versus baryo-chemical potential representing chemical freeze-out tend to merge with the phase boundary between partonic and hadronic media predicted by the lattice QCD calculations $[1,2,3]$. At higher $\mu_{B}$ however, the divergence of the two curves suggests the existence of a dense yet hadronic medium, which can be probed by nuclear collisions at beam energies between AGS and top SPS. Further insight into the dynamics of nucleus-nucleus collisions is obtained by hydrodynamical calculations [4] of the trajectories in the $\mathrm{T}-\mu_{B}$ plane. According to these results, the deconfinement phase border is first reached around $10 \mathrm{AGeV}$ beam energy; at $30 \mathrm{AGeV}$, the trajectory passes near the critical endpoint. At top SPS energy, the system evolves towards the low $\mu_{B}$ region of the phase diagram. These calculations suggest that phenomena connected with deconfinement as a result of a 1st order phase transition and the critical point are best studied using beam energies available at SPS.

The NA49 experiment obtained numerous interesting results related to both the onset of deconfinement and to the critical point [5, 6, 7]. Indications for the onset of deconfinement are seen in the energy dependence of the pion yield per number of participating nucleons (kink), of the $\left\langle K^{+}\right\rangle /\left\langle\pi^{+}\right\rangle$ratio (horn), and of the mean transverse mass or inverse slope parameters of $m_{T}$ spectra (step) in central $\mathrm{Pb}+\mathrm{Pb}$ collisions for the beam energy of $30 \mathrm{AGeV}$.

Both the theoretical and experimental results outlined above have motivated the continuation of the heavy ion program at the CERN SPS which will be performed by the NA61/SHINE experiment. The main goal of the ion program of this experiment is to perform systematic search for the critical point of strongly interacting matter and study in detail the properties of the onset of deconfinement. These physics goals will be pursued by performing a two-dimensional scan in the whole SPS energy range from $10 \mathrm{~A}$ to $158 \mathrm{~A} \mathrm{GeV}$ with light and intermediate mass nuclei $(\mathrm{p}+\mathrm{p}, \mathrm{p}+\mathrm{Pb}, \mathrm{B}+\mathrm{C}, \mathrm{Ar}+\mathrm{Ca}$, $\mathrm{Xe}+\mathrm{La}$ ). NA61/SHINE intends to register of $2 \times 10^{6}$ central events for each reacting system and energy. In order to accomplish physics goals of the new experimental program, several upgrades of the detector apparatus inherited from NA49 are performed and foreseen. In 2007, a forward Time-of-Flight was constructed in order to extend the acceptance for particles with $p<3 \mathrm{GeV} / \mathrm{c}$; in 2008, a new TPC readout and DAQ system with about $70 \mathrm{~Hz}$ readout frequency were installed and tested. These modifications increased the event rate by a factor of about 10. In 2010 first test of the assembled Projectile Spectator Detector have been performed. This detector will provide a single nucleon energy resolution necessary for the fluctuation studies essential in search for the critical end-point. In addition a new low mass helium beam pipe will reduce the delta electron background by a factor of 10 . As it was mentioned the NA61/SHINE physics program requires low and intermediate mass ion beams, however, only $\mathrm{Pb}$ beams will be available in the coming years. Thus the use of a secondary ion beams derived from the fragmentation products of primary $\mathrm{Pb}$ ions is proposed [8]. The experimental program was started in 2009 with measurements of $\mathrm{p}+\mathrm{p}$ interactions. Low mass ion $(\mathrm{B}+\mathrm{C})$ runs are foreseen for 2011, while $\mathrm{Ar}+\mathrm{Ca}$ and $\mathrm{Xe}+\mathrm{La}$ interactions are planned for 2013 and 2014, respectively. 
A more comprehensive description of the NA61/SHINE experiment and it's physics program can be found in $[9,10]$.

\section{References}

[1] A. Andronic, P. Braun-Munzinger, J. Stachel, Nucl. Phys. A 772, (2006) 167.

[2] P. Braun-Munzinger, Nucl. Phys. A 681, 119c-123c (2001).

[3] A. Andronic, P. Braun-Munzinger, J. Stachel, arXiv:09114931 [nucl-th].

[4] Yu. B. Ivanov, V. N. Russkikh, and V. D. Toneev, Phys. Rev. C 73, 044904 (2006).

[5] M. Gaździcki and M. I. Gorenstein, Acta Phys. Pol. B 30, 2705 (1999).

[6] M. I. Gorenstain, M. Gaździcki and K. A. Bugaev, Phys. Lett. B 567, 175 (2003).

[7] M. Gaździcki at al., Braz. J. Phys. 34, 322 (2004).

[8] N. Abgrall at al. [The NA49 Collaboration], CERN-SPSC-2007-033, CERN-SPSC-P-330.

[9] K. Grebieszkow for the NA49 and NA61 Collaborations, Acta Phys. Pol. B 41, (2010) 427.

[10] G. Stefanek for the NA61 Collaboration, PoS(CPOD 2009) 049. 